\title{
Transfissural Approach for Laparoscopic Resection of a Deep Segment 8 Lesion in Contact with the Hepatocaval Confluence
}

\author{
Satoshi Ogiso, MD, PhD, FACS, Satoru Seo, MD, PhD, Takamichi Ishii, MD, PhD, Tomoaki Yoh, MD, PhD, \\ Yukinori Koyama, MD, PhD, Ken Fukumitsu, MD, PhD, and Kojiro Taura, MD, PhD
}

Division of Hepato-Biliary-Pancreatic Surgery and Transplantation, Department of Surgery, Graduate School of Medicine, Kyoto University, Kyoto, Japan

\section{BACKGROUND}

Patients with liver lesions contacting the hepatocaval confluence are non-optimal candidates for laparoscopy. The resection poses a risk of massive bleeding as well as fatal gas embolism in the case of injury to major hepatic veins or the vena cava. Although meticulous dissection is needed to detach lesions from the hepatocaval confluence, laparoscopy has significant limitations in terms of viewing and accessing the paradome area.

\section{METHODS}

Widely opening the midplane of the liver along the middle hepatic vein (MHV), termed the "transfissural approach," makes the hepatocaval confluence easily accessible for laparoscopy and provides a good surgical field for safe detachment of the lesion from major hepatic veins and the vena cava.

\section{RESULTS}

A 74-year-old man underwent laparoscopic segment 8 resection for a 55-mm colorectal liver metastatic lesion in segment 8 contacting the right hepatic vein (RHV) and the vena cava. The MHV was detected by transection of the midplane from the caudal side and was followed cranially. Complete transection of the ventral parenchyma of the MHV facilitated dorsal dissection of the lesion. After transection of the caudal and left-side borders of segment 8 and division of the segment 8 Glissonean pedicle, tumorinfiltrating branches of the MHV and RHV origins were divided, and the lesion was safely detached from the vena cava and the RHV origin.

\section{DISCUSSION}

The transfissural approach improves access to the deep part of the paradome area and increases the safety of laparoscopic segment 8 resection for lesions contacting the hepatocaval confluence.

DISCLOSURES Satoshi Ogiso, Satoru Seo, Takamichi Ishii, Tomoaki Yoh, Yukinori Koyama, Ken Fukumitsu, and Kojiro Taura have no conflicts of interest.

Publisher's Note Springer Nature remains neutral with regard to jurisdictional claims in published maps and institutional affiliations.
Electronic supplementary material The online version of this article (https://doi.org/10.1245/s10434-020-09309-5) contains supplementary material, which is available to authorized users.
(C) Society of Surgical Oncology 2020

First Received: 9 July 2020;

Published Online: 9 November 2020

S. Seo, MD, PhD

e-mail: rutosa@kuhp.kyoto-u.ac.jp 